\title{
Impact of adjuvant therapeutic surgery on the health-related quality of life of pulmonary tuberculosis patients
}

\author{
Pau Benito ${ }^{1,2}$, Sergo Vashakidze ${ }^{3}$, Shota Gogishvili ${ }^{3}$ Keti Nikolaishvili ${ }^{3}$, \\ Albert Despuig ${ }^{1,4}$, Nestan Tukvadze ${ }^{3}$, Natalia Shubladze ${ }^{3}$, Zaza Avaliani $^{3}$ and \\ Cristina Vilaplana $\mathbb{1}^{1,4}$
}

Affiliations: ${ }^{1}$ Experimental Tuberculosis Unit (UTE), Fundació Institut Germans Trias i Pujol (IGTP), Universitat Autònoma de Barcelona (UAB), Badalona, Spain. ${ }^{2}$ Faculty of Health and Life Sciences (FCSV), Universitat Pompeu Fabra and UAB, Barcelona, Spain. ${ }^{3}$ National Center for Tuberculosis and Lung Diseases, Tbilisi, Georgia. ${ }^{4}$ Centro de Investigación Biomédica en Red de Enfermedades Respiratorias (CIBERES), Madrid, Spain.

Correspondence: Cristina Vilaplana, UTE, Fundació IGTP, Crtra. de Can Ruti, Camí de les Escoles, Edifici Mar, s/n, 08916, Badalona, Barcelona, Spain. E-mail: cvilaplanađigtp.cat

ABSTRACT This study aimed to determine the health-related quality of life (HRQoL) of patients with pulmonary tuberculosis (TB) and to assess its change after a therapeutic surgical procedure. In this scenario, the purpose was to elucidate and quantify the effect of various demographic, epidemiological, clinical, surgical and psychosocial details on this variable.

A prospective cohort of 40 patients undergoing therapeutic surgery for pulmonary TB (Study of Human Tuberculosis Lesions (SH-TBL) cohort) was recruited in Tbilisi, Georgia, between 2016 and 2018. HRQoL was assessed by administering the St George's Respiratory Questionnaire (SGRQ) and a novel psychosocial questionnaire, the BCN-Q, both at baseline and at 6 months post-surgery.

A statistically and clinically significant improvement in the SGRQ total score was observed at follow-up, although it did not reach the values found for the healthy population. The differences between time points were statistically significant for the following groups: women, age $<40$ years, body mass index $\geqslant 20 \mathrm{~kg} \cdot \mathrm{m}^{-2}$, nonsmokers, drug-susceptible and drug-resistant participants, both new and relapsed patients, early culture negativisation, cases with a single lesion, either lesions $<35 \mathrm{~mm}$ or $\geqslant 35 \mathrm{~mm}$, and lesion, lobe and lung resections.

The analysis of BCN-Q together with the SGRQ showed that several of its items, such as marital status, living conditions, nutrition, employment, external support, certain attitudes towards the healthcare system, emotional burden and sleep troubles, can impact HRQoL.

These results highlight the benefit of adjuvant therapeutic surgery for pulmonary TB in selected patients in terms of HRQoL and suggest that a comprehensive approach including demographic, epidemiological, clinical and psychosocial variables may more accurately predict TB evolution and prognosis.

@ERSpublications

Adjuvant therapeutic surgery in selected pulmonary TB patients improves their health-related quality of life. Impact of psychosocial variables on HRQoL may be assessed using a newly developed questionnaire, namely BCN-Q. https://bit.ly/2A169rR

Cite this article as: Benito P, Vashakidze S, Gogishvili S, et al. Impact of adjuvant therapeutic surgery on the health-related quality of life of pulmonary tuberculosis patients. ERJ Open Res 2020; 6: 00083-2020 [https://doi.org/10.1183/23120541.00083-2020].

This article has supplementary material available from openres.ersjournals.com

The work presented here is part of the SH-TBL (ClinicalTrials.gov: NCT02715271).

Received: 21 Feb 2020 | Accepted after revision: 29 May 2020

Copyright $\odot$ ERS 2020. This article is open access and distributed under the terms of the Creative Commons Attribution Non-Commercial Licence 4.0. 


\section{Introduction}

One of the most important tuberculosis (TB) current challenges is the global spread of multidrug- (MDR) and extensively drug-resistant (XDR) forms, the management of which is highly complex as only a few therapeutic options are available $[1,2]$. The World Health Organization (WHO) consolidated guidelines on MDR-TB treatment include elective partial lung resection as a possible adjunctive treatment alongside a recommended pharmacological scheme [3]. Therapeutic surgery is prescribed to manage complicated cases of TB (even for selected drug-sensitive (DS) cases), or those in which medical treatment is failing [1]. In countries of the former Soviet Union, surgery has remained a relatively widespread therapeutic option.

Addressing MDR-TB is one of the End TB Strategy targets, as well as tackling the social determinants underlying $\mathrm{TB}$, including poverty, crowded and poorly ventilated living and working conditions and malnutrition, amongst others. The End TB Strategy also contemplates the social burden of TB, which encompasses stigmatisation, interruption of studies, loss of employment and others, and stresses the need for social protection measures [4].

\section{TABLE 1 Main characteristics of the study cohort}

Characteristics of participants

Sex

Male

Age years

BMI $\mathrm{kg} \cdot \mathrm{m}^{-2}$

Smoking status

Yes

Comorbidities

Psychiatric diseases

Diabetes

COPD

Severe renal disease

HIV infection

HCV infection

Cirrhosis

Others (HBV)

Medication

NSAIDs

Statins

Oral anticoagulants

Heparin

Corticosteroids

Drug susceptibility

Drug-susceptible

MDR

XDR

History of previous treatment

New patient

Relapse patient

Time to culture negativisation

Indication for surgery

Still lesions in chest X-ray

Clinically not cured

Haemoptysis

Number of lesions

Single lesion

Size of lesion

$\geqslant 35 \mathrm{~mm}$

Type of surgery

Lesion resection

Lobe or lung resection
Study population $(n=40$ )

$19(47.5)$

$33.5(15-61)$

$22.5(16.5-32.0)$

15 (37.5)

0 (0)

3 (7.5)

$1(2.5)$

$0(0)$

$0(0)$

$6(15.0)$

$0(0)$

$2(5.0)$

$0(0)$

$0(0)$

$0(0)$

$0(0)$

$2(5.0)$

15 (37.5)

18 (45.0)

7 (17.5)

27 (67.5)

13 (32.5)

$2.4(1-10)$

$40(100)$

$4(10.0)$

$2(5.0)$

$32(80.0)$

14 (35.9)

15 (37.5)

Data are presented as $\mathrm{n}(\%)$ or mean (range). BMI: body mass index; HCV: hepatitis $\mathrm{C}$ virus; HBV: hepatitis $B$ virus; NSAID: non-steroidal anti-inflammatory drug; MDR: multidrug resistant; XDR: extensively drug-resistant. 
The aim of this study was to determine the impact of adjunctive therapeutic surgery on the health-related quality of life (HRQoL) of pulmonary TB patients, as well as to assess the role of various clinical and epidemiological characteristics of participants. Furthermore, this study aimed to determine influential psychosocial items collected from a novel questionnaire employed in this cohort.

\section{Methods}

\section{Study design}

This study followed prospectively all patients undergoing therapeutic surgery for pulmonary TB at the National Center for Tuberculosis and Lung Diseases (NCTLD, Tbilisi, Georgia) and consenting to donate samples and data for the study, recruited between May 2016 and May 2018 ( $\mathrm{n}=40$, Study of Human Tuberculosis Lesions (SH-TBL) cohort).

The NCTLD internal committee was responsible for indication of surgery. Decision was made on the basis of persistent radiological TB lesions in chest radiography (all the participants) and/or incessant clinical manifestations such as fatigue, cough, fever or even haemoptysis within the last 4 weeks from the surgery date, despite the fact of having completed the corresponding pharmacological anti-TB regimen (table 1). All patients were microbiologically cured prior to surgery according to WHO criteria (negativisation for both sputum culture and acid-fast bacilli smear after finishing the pharmacological treatment scheme).

For each patient, demographic, clinical and TB-related information were obtained prior to surgery. Two HRQoL questionnaires, the Saint George's Respiratory Questionnaire (SGRQ) and the BCN Questionnaire (BCN-Q), were administered to participants before and 6 months after surgery.

\section{Data work}

OpenClinica (S4 Research) was the interface of the electronic case report form (eCRF). Content from the eCRF was transferred into a Microsoft Office Excel 2010 spreadsheet, in which the SGRQ symptoms, activity, impacts and total scores were calculated. GraphPad Prism version 8.2.0.435 was used to elaborate the graphs and the statistical analysis.

\section{Statistical analysis}

Data are shown as mean \pm SEM. As a result of effectuating the Shapiro-Wilk test, normal distribution could not be assumed for SGRQ total score. A Wilcoxon matched-pairs signed-rank test was conducted when comparing time points and a Mann-Whitney U-test was used both when comparing groups in each time point and when determining associations between answers to BCN-Q and SGRQ total score. A Kruskal-Wallis test was applied to comparisons between more than two groups. A Chi-squared test, or Fisher's exact test when there were only two possible answers, were performed to compare answers to

FIGURE 1 St George's Respiratory Questionnaire (SGRQ) score change according to time point. Graphs show the participants' score for each of the components of the questionnaire la: symptoms, b: activity and c: impacts), as well as their overall total score (d) at both time-points.
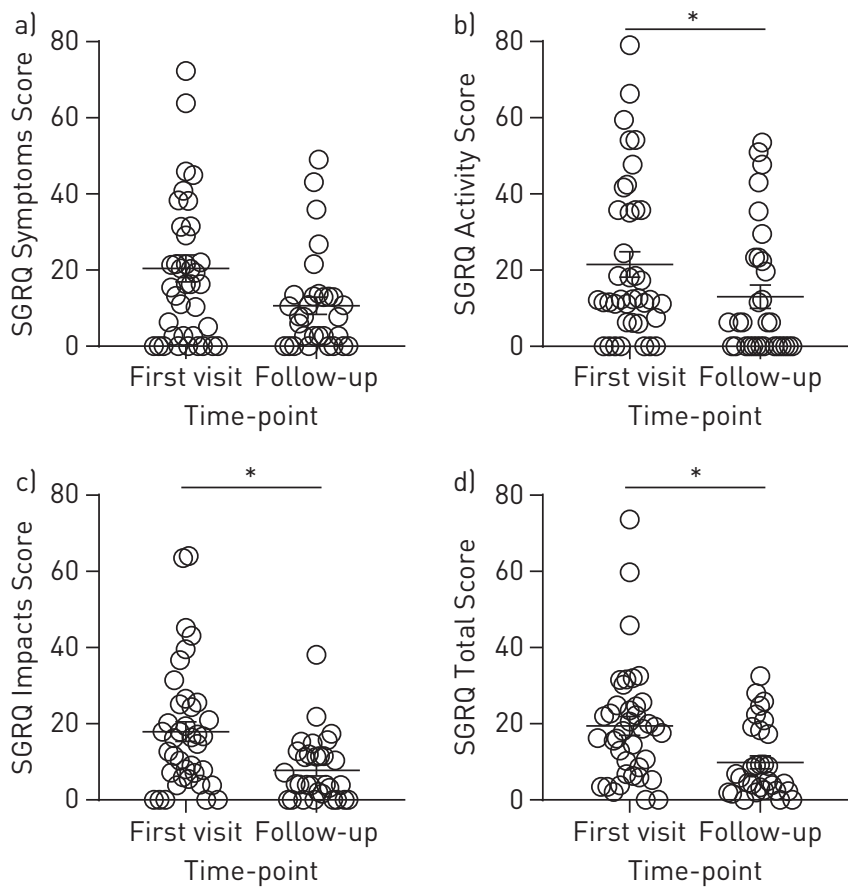
questions in BCN-Q between time points. All p-values were two-tailed, with the statistical significance at $\mathrm{p}<0.05$.

\section{Ethics}

This paper is part of the SH-TBL project (ClinicalTrials.gov: NCT02715271), led by the Experimental Tuberculosis Unit and conducted in collaboration with the NCTLD. The protocol and research methodology, as well as associated documents (informed consent sheet, informed consent form) were reviewed and approved by both ethics committees at the NCTLD (IRB00007705 NCTLD Georgia \#1, IORG0006411) and the Germans Trias i Pujol Research Institute (EC: PI-16-171). Written informed consent was obtained from all study participants before they were enrolled.

\section{Results}

\section{HRQoL baseline versus follow-up}

The $87.5 \%$ of questionnaires were answered: 39 out of 40 at baseline and 31 out of 40 at 6 months after surgery. A few answers were missed for some patients. SGRQ score scale is $0-100$, with higher scores indicating a worse status [5-7]. After surgery, a score decrease of around 10 units was observed for all the components (symptoms, activity and impacts) and for the total score, differences being statistically significant (except for the symptoms score) (figure 1 and table S1).

\section{Demographic and clinical characteristics}

40 patients (19 males and 21 females) were included. Mean age was 33.5 years and mean body mass index (BMI) was $22.5 \mathrm{~kg} \cdot \mathrm{m}^{-2}$. Overall, 15 patients were smokers (37.5\%). Among their comorbidities and relevant medication, it stood out that six were infected with hepatitis $\mathrm{C}$ virus and two with hepatitis $\mathrm{B}$ virus (although none had cirrhosis) and two were taking corticosteroids (table 1 and table S1).

Women significantly improved their SGRQ total score after surgery and maintained lower scores than men at both time points. Unlike patients aged $\geqslant 40$ years, the youngest obtained a statistically significant score decrease after surgery. Participants with a BMI $<20 \mathrm{~kg} \cdot \mathrm{m}^{-2}$ had lower mean scores than those with a BMI $\geqslant 20 \mathrm{~kg} \cdot \mathrm{m}^{-2}$ but differences after surgery were statistically significant only in the latter. Nonsmokers had lower scores than smokers and reached a statistically significant score improvement after surgery (figure 2 and table S2).

\section{TB and surgery-related information}

All patients had pulmonary TB: 15 DS, 18 MDR and 7 XDR-TB. For DS participants, the commonest pharmacological anti-TB treatment received prior to surgery was a 6-month scheme with moxifloxacin, rifampicin, pyrazinamide and ethambutol. Two of them received isoniazid instead of moxifloxacin.

FIGURE 2 St George's Respiratory Questionnaire (SGRQ) total score change according to clinical and demographic characteristics of participants and time point: al sex, b) age, cl body mass index (BMI) and d) smoking status. Circular and square symbols correspond to data from the first visit and follow-up, respectively.
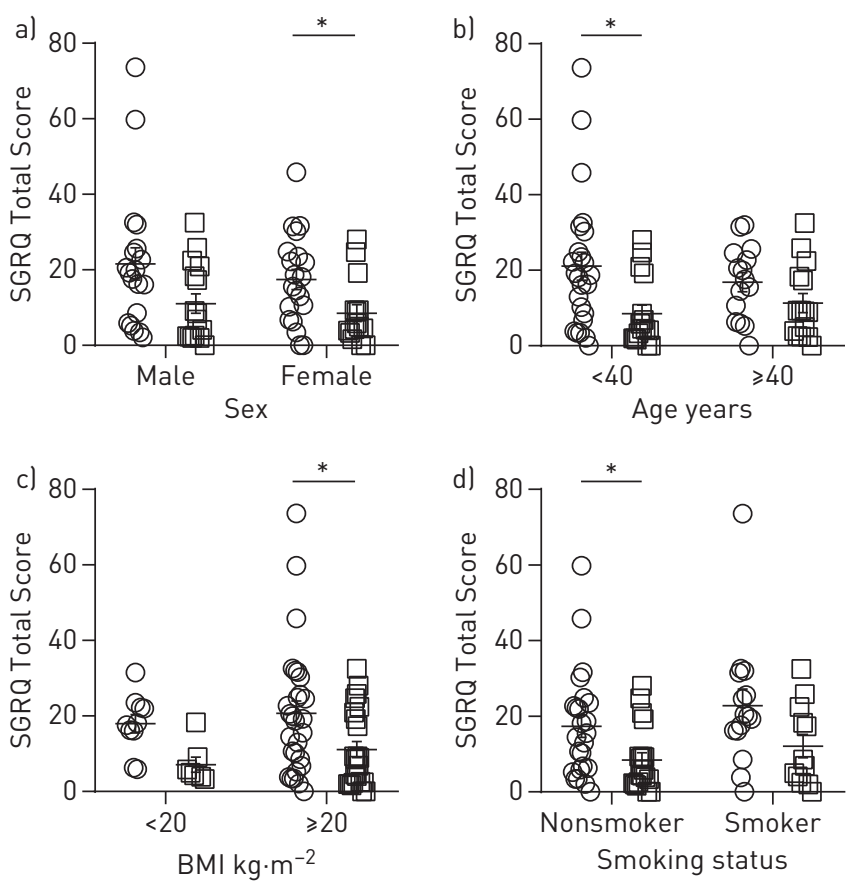
For MDR and XDR-TB patients, treatment was extended to 8-10 months and consisted of individualised combinations of 3-6 drugs, with pyrazinamide, capreomycin, respiratory quinolones (levofloxacin, moxifloxacin), cycloserin, PAS, linezolid and clofazimine among the most-employed anti-TB agents. Nearly one-third were relapsed patients. Lesions, typically unique $(80.0 \%)$, were of very variable sizes and in most cases led to a lesion resection surgery (62.5\%) (table 1).

Both DS and drug-resistant (when analysing MDR and XDR jointly) participants had statistically significant score improvements after surgery. New patients scored significantly better than relapse patients before surgery $(\mathrm{p}=0.0338)$ but, as both new and relapse patients significantly decreased their score after surgery, differences at the follow-up were minimal. Regardless of statistical significance being only achieved for early culture converters ( $\leqslant 2$ months), improvement for late culture converters ( $>2$ months) almost reached the threshold for statistical significance (figure 3 and table S3).

Unlike patients with multiple lesions, participants with a single lesion significantly decreased their score after surgery, regardless of the lesions being categorised as big $(\geqslant 35 \mathrm{~mm})$ or small $(<35 \mathrm{~mm})$ and the surgical procedure being a lesion, lobe or entire lung resection (figure 3 and table S3).

\section{Description of answers to $B C N-Q$}

BCN-Q focuses on the psychosocial reality of TB patients. Approximately two-thirds of participants had a partner at both time points. Baseline unemployment rate was $59.0 \%$, dropping to $37.9 \%$ at follow-up. The majority of participants lived in their own house or apartment, in most cases with their family. They were predominantly living with 3 to 6 cohabitants, two of them providing financially in most cases.

The majority of participants had not asked for any kind of assistance. More than a half always received family financial support, but participants not needing it increased from $5.1 \%$ to $19.4 \%$ after surgery. Around $60 \%$ did not have to take care of anybody, the rest having to take care of children in most cases. Participants with the lowest meat, fish and egg intake dropped from $23.1 \%$ to $3.2 \%$ after surgery and the same occurred for patients eating dairy products once a week or never, who were reduced from $28.2 \%$ to $13.0 \%$.

The vast majority of patients knew what their condition was and thought it was very serious or at least serious. Most of them did not know how they were infected. Patients were mainly afraid of infecting family members but their fear was reduced at follow-up. Half of them had met more than 10 people who had had TB. Most patients sought assistance shortly after feeling unwell. Practically none thought it was a disgrace for the family to have a member with TB. In this regard, they did not generally hide their condition, especially at follow-up. Patients considering TB as a curse decreased from $37.1 \%$ to $20.7 \%$ after surgery. Less than a quarter resorted to traditional medicine.

Most patients considered they always had the support from their families and the family encouraged them a lot in taking their medicine daily. Friends or acquaintances generally did not desert them. The majority of participants said they absolutely trusted both their physician and, to a lesser extent, the other staff members. Almost all patients thought all staff or at least some of them were being friendly.

The immense majority considered their condition had changed their life and/or personality. A quarter claimed to have lost their job because of TB. Most participants had never been incarcerated. Patients experiencing trouble sleeping significantly dropped between time points $(46.2 \%$ versus $16.6 \%, \mathrm{p}=0.0361$ ) Less than half of patients thought TB had influenced their career trajectory and less than $10 \%$ considered that it diminished their chances to marry. Thinking of abandoning treatment was rather infrequent. A total of 31 out of 39 participants at baseline and 14 out of 31 at follow-up reported an emotional burden, which equates to $34.3 \%$ decrease between time points. Only 1 in 10 thought they were not going to fully recover. Among MDR/XDR patients, the commonest sequelae were dysesthesias, which significantly decreased at follow-up (16 out of 24 versus 6 out of 16, $\mathrm{p}=0.0471$ ).

Table S4 in the supplementary material provides a complete description of patients' responses to the questionnaire.

\section{Correlation between $B C N-Q$ and SGRQ}

Some of the aforementioned questions were analysed in relation to the HRQoL data obtained using the SGRQ (figure 4 and table S5). Patients without a partner showed a greater score decrease between time points when compared to patients who were married or had a partner but only differences in the latter were statistically significant. Patients living with five or more cohabitants significantly improved after surgery and maintained lower scores than those living with four or fewer cohabitants.

Employed participants maintained better scores than those unemployed. Patients who had lost their job due to TB had worse scores than participants who had not lost it, especially before surgery $(\mathrm{p}=0.0477)$. 

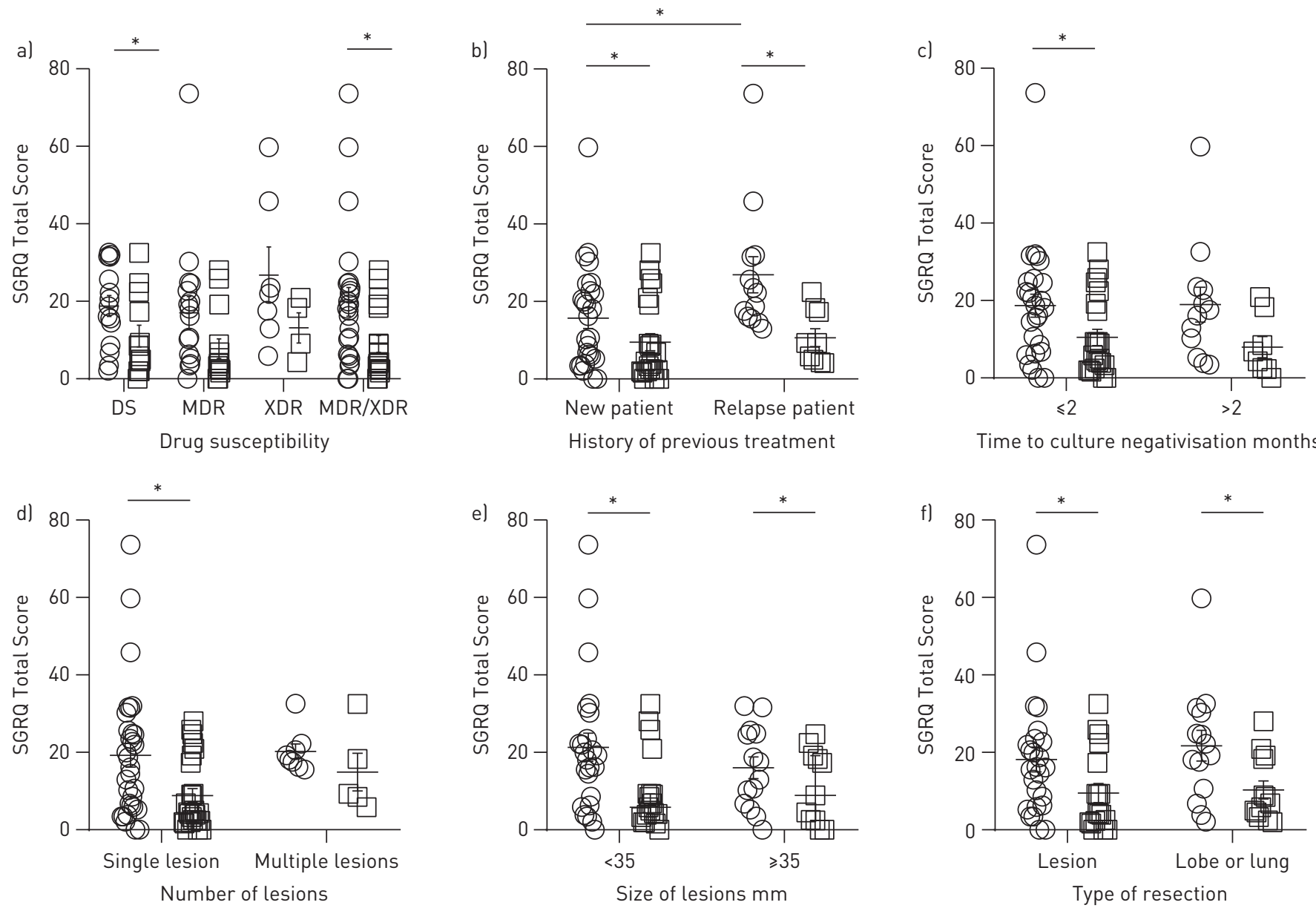

FIGURE 3 St George's Respiratory Questionnaire (SGRQ) total score change according to tuberculosis and surgery-related information of participants and time point: a) drug susceptibility, b) history of previous treatment, c) time to culture negativisation, d) number and e) size of lesions and f) type of resection. Circular and square symbols correspond to data from the first visit and follow-up, respectively. DS: drug-susceptible; MDR: multidrug resistant; XDR: extensively drug-resistant.

A statistically significant score decrease was observed both in patients with more financial support and in those not having to take care of anybody.

Participants with the lowest protein intake had the worst scores at both the baseline (meat, fish, eggs and dairy products) and follow-up (all but dairy products). Those not turning to traditional/natural medicines significantly improved after surgery and maintained lower scores than the antagonistic group. Trouble sleeping was associated with higher mean scores at both the baseline $(\mathrm{p}=0.0004)$ and follow-up.

Patients reporting emotional burden had a higher baseline mean score than their opposites $(p=0.0133)$ but significantly improved their score after surgery. MDR/XDR participants suffering pharmacological sequelae had higher baseline scores than those without sequelae but the situation was inverted after surgery as they significantly improved after surgery.

\section{Discussion}

\section{Impact of surgery on HRQoL}

Few studies have utilised standardised quality of life (QoL) instruments in patients with $\mathrm{TB}$. Consequently, there are many gaps in understanding QoL among TB patients, and how it changes with treatment and cure [8]. There is no consensus regarding a gold-standard HRQoL questionnaire for TB. WHO QoL, SF-36 and SGRQ are examples of the most commonly employed instruments, none of them being TB-specific [9].

The SGRQ Manual estimated the expected mean scores for healthy subjects as 12, 9, 2 and 6 for symptoms, activity, impacts and total score, respectively [5]. Another study with a larger sample calculated the mean normative values for the general population as 9.67, 13.40, 4.74 and 8.41, respectively [10]. The estimated threshold for a clinically significant change in health status is consistently around four units [11]. 

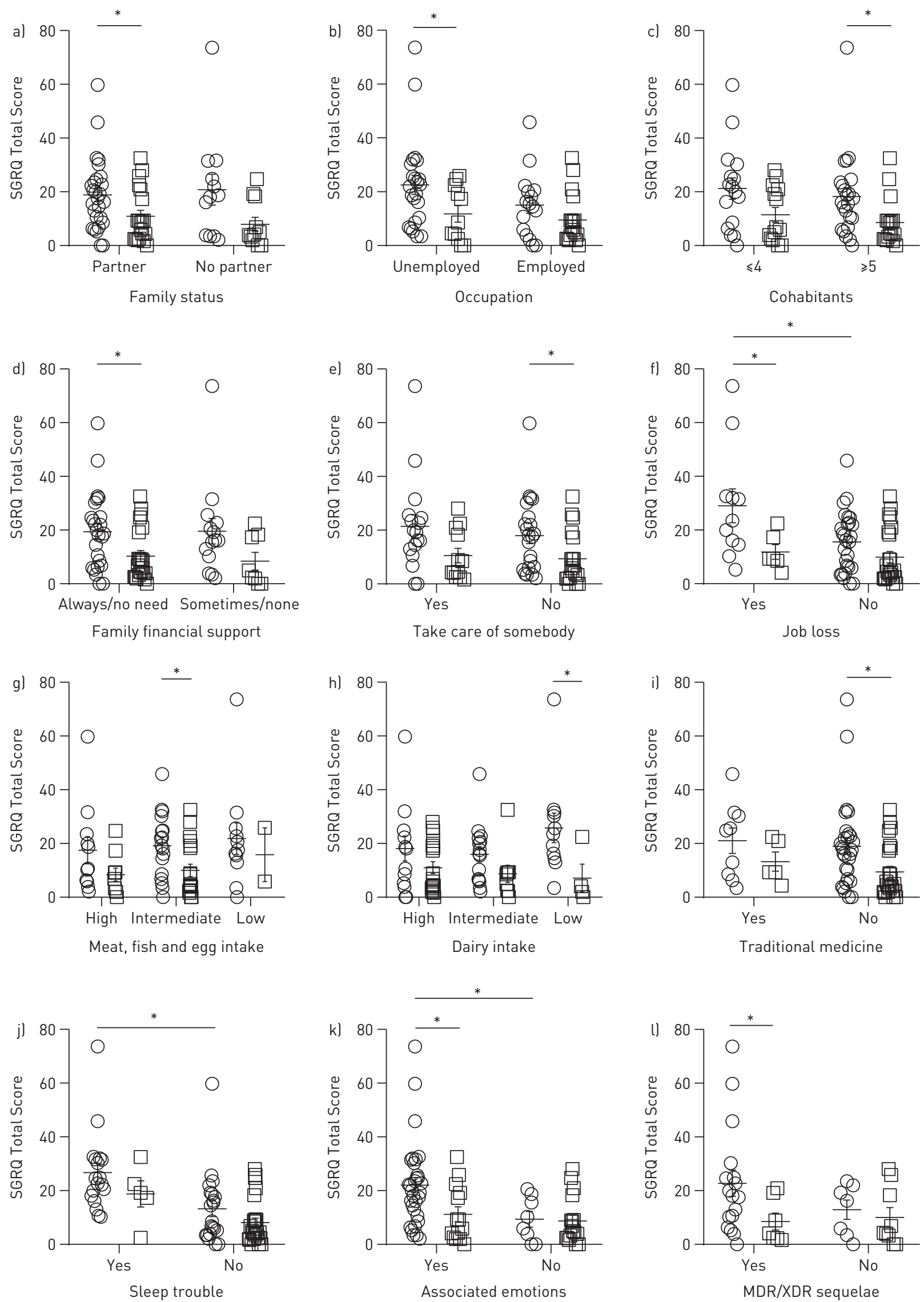
FIGURE 4 St George's Respiratory Questionnaire (SGRQ) total score change according to answers to the BCN-Q and time point. Graphs show the score differences depending on al family or marital status, b) occupation, c) number of cohabitants, d) family financial support, el need to take care of somebody, fl job loss due to tuberculosis, gl meat, fish and eggs intake, hl dairy products intake, il use of traditional medicine, jl sleep troubles, k) emotional burden and () multidrug resistant (MDR)/extensively drug-resistant (XDR) sequelae. Circular and square symbols correspond to data from the first visit and follow-up, respectively.

The results of our study revealed an improvement of around 10 units in all dimensions of the SGRQ at follow-up, thus showing a clinically significant benefit of adjuvant therapeutic surgery for pulmonary TB in selected patients in terms of HRQoL. Mean scores for symptoms and activity were in the range of the reference values for the general population. Impacts and total scores remained above them. Residual differences are apparently not clinically significant if considering the aforementioned four-unit threshold.

These findings are consistent with a review of nonsurgical studies by Brown et al. [9], which claimed that active TB was associated with a lower health status and treatment was related to improvements in HRQoL, although residual deficits persisted after it. Very recently, a cross-sectional study in successfully treated drug-resistant TB patients showed better SGRQ scores among those undergoing adjunctive surgery [12].

Despite this study being valuable as a formal assessment of HRQoL change after adjunctive surgery for both DS and drug-resistant TB patients, further studies comparing adjunctive surgery with other approaches, such as continuing the same or another pharmacological treatment scheme, should be performed to thoroughly determine if the HRQoL improvement is independently related to the surgery.

\section{Influence of individual characteristics in HRQOL}

The worldwide male-to-female case notification ratio is 1.7 , the reasons for this observed sex bias remaining controversial [13]. Nonetheless, examination of time to sputum conversion and type of necrosis in surgical samples suggested that TB course could be worse in women [14]. In our study, women's scores were slightly better both before and after the surgery.

Young participants significantly improved after surgery. Even though the maximum age in our cohort was 61 years, age-related physiological changes, including loss of immunity, might partly explain the poorer response of the oldest [15].

Weight loss is a typical clinical sign of TB disease. Existing evidence claims that a poorer nutritional status leads to secondary immunodeficiency [16], a radiological increase in the extent of disease [17] and increased mortality [18]. In accordance, and despite the fact that participants with a BMI $<20 \mathrm{~kg} \cdot \mathrm{m}^{-2}$ had lower mean scores than those with a BMI $\geqslant 20 \mathrm{~kg} \cdot \mathrm{m}^{-2}$, only the latter achieved a significant improvement after surgery.

Our study found that smoking status is a predictor of HRQoL evolution during TB treatment, which is consistent with abundant evidence highlighting its role as an indicator of torpid evolution [19].

Observational studies have stated high treatment success rates, a trend towards improved outcomes, and acceptable morbidity for adjunctive surgery as a treatment for MDR/XDR tuberculosis [20]. Our study suggests considering the need for future studies with larger samples to elucidate the potential role of adjuvant surgery in the treatment of DS-TB patients in whom medical treatment has been insufficient.

In our cohort, baseline HRQoL was significantly poorer in relapse patients, which could be attributed to sequelae from previous TB episodes. Differences were attenuated at follow-up, suggesting that surgery can be helpful in some of these patients.

Early culture converters achieved a statistically significant improvement in HRQoL after the surgery, but late converters almost reached the threshold for statistical significance. Therefore, it cannot be said that time to culture conversion determine the impact of surgery on HRQoL. Our results suggest that patients with a single lesion are more likely to improve their HRQoL after surgery than patients with multiple lesions, irrespective of lesion size and type of procedure. It could be related to offering a simpler surgical approach and an easier control of disease focus.

\section{$B C N-Q$ : the psychosocial perspective and its relationship with the HRQoL results}

None of the traditional tools used to evaluate HRQoL in TB are TB-specific instruments [9]. Recently, at least two disease-specific HRQoL instruments, namely FACIT-TB [21] and QLICD-TB [22], have been developed. Applying a TB-specific perspective may be relevant as the social, economic and emotional burden of TB are well known. This motivated us to design and administer an additional questionnaire, namely the BCN-Q, which focuses on psychosocial aspects and the perspective of $\mathrm{TB}$ patients themselves, and to determine which variables translated to statistically significant changes in HRQoL after surgery. 
Loss of employment prior to surgery was associated with a worse baseline HRQoL. Studies emphasise the perceived risk of infecting others and the feeling of lack of energy among job loss causes [23, 24]. Unemployment rate decreased in our cohort after surgery. As such, we hypothesise its role in increasing patients' fitness and in reducing employer's fear of contagion.

Protein malnutrition correlates with poor tuberculosis treatment outcomes, including treatment failure, relapse and death $[25,26]$. Accordingly, participants with the lowest protein intake in our cohort had a worse HRQoL at both baseline (meat, fish, eggs and dairy products) and follow-up (all but dairy products). Patients with poor protein intake clearly dropped after surgery. Anti-TB treatment is known to improve appetite loss, nutrient malabsorption and metabolism alterations provoked by TB disease [25, 27, 28]. Hence, our findings are similar to those described in patients receiving pharmacological treatment for TB.

Crowded living conditions are associated with an increased risk of infection [29] and a poorer disease outcome [30]. In our cohort, participants who were married or living as a couple, and patients living with five or more cohabitants, improved significantly after surgery, probably because many cohabitants assumed the role of carers. Family and close relationships are known to positively affect illness management [31].

Preventing families from experiencing catastrophic costs due to TB is an End TB Strategy goal [32]. Previous studies have shown the relevance of financial risk protection interventions [33]. Differences in our cohort are of small magnitude but only patients who always received family financial support or who did not need it and those not having to take care of anybody achieved a significant improvement after surgery.

In our cohort, resorting to traditional medicine limited the HRQoL improvement. A delay in seeking medical assistance, a reduced treatment adherence and a perception of poor response to it could play a role on this association.

Psychological distress accompanies many diseases. Our participants were asked how they were feeling. Nearly $80 \%$ reported some kind of emotional burden at baseline, decreasing to $45 \%$ at follow-up, which are similar results to those found by Tola et al. [34]. In our cohort, the association of emotional burden with a worse HRQoL (especially at baseline) together with the reduction in the number of patients facing emotional burden at follow-up reinforce the positive impact of surgery and support the two-way relationship between mental and physical health.

Respiratory diseases, particularly COPD, are associated with poor sleep quality [35], which negatively affects HRQoL [35, 36] and patient functionality [37]. In our cohort, 46\% of participants suffered sleep disturbances at baseline, with their HRQoL being much worse. After surgery, participants with sleep troubles still had a worse HRQoL but represented only $17 \%$ of the cohort. The relationship between poor sleep quality and HRQoL might be bidirectional if, as reported for COPD, disease severity correlates with sleep troubles [38].

Pharmacological sequelae among MDR/XDR-TB patients were associated with a much worse baseline HRQoL. The interpretation is not evident as SGRQ specifically inquiries about respiratory disturbances. Reduced treatment adherence because of adverse events could partly explain it. Moreover, adverse events might influence how participants rate their HRQoL. Existing evidence on this issue shows contrasting results $[39,40]$.

\section{Conclusions}

This study provides evidence for the benefit of therapeutic surgery for pulmonary TB in selected patients, as measured both quantitatively using the SGRQ score and qualitatively using a newly developed HRQoL questionnaire. Sex, age, BMI, smoking status, drug susceptibility, history of previous treatment and the nature of the lesions should be taken into account as they influence the outcome of surgery. Our study also highlights that a comprehensive approach to $\mathrm{TB}$, and particularly to its HRQoL, must include a psychosocial perspective. The results of the BCN-Q suggest its utility for evaluating the psychosocial burden of TB, which should be validated in other TB cohorts.

Acknowledgements: We thank the patients who agreed to be involved in this study.

Conflict of interest: P. Benito has nothing to disclose. S. Vashakidze has nothing to disclose. S. Gogishvili has nothing to disclose. K. Nikolaishvili has nothing to disclose. A. Despuig reports grants from Spanish Government-FEDER Funds and the CIBER Enfermedades Respiratorias Network (CIBERES), and personal fees from Agència de Gestió d'Ajuts Universitaris i de Recerca AGAUR, during the conduct of the study. N. Tukvadze has nothing to disclose. N. Shubladze has nothing to disclose. Z. Avaliani has nothing to disclose. C. Vilaplana reports personal fees from Spanish Government-FEDER Contratos Miguel Servet, and grants from Spanish Government-FEDER, CIBERES), grants from the Spanish Society of Pneumology and Thoracic Surgery (SEPAR), during the conduct of the study. 
Support statement: This study was funded by the Spanish Government-FEDER Funds through CV contracts CP13/ 00174, CPII14/00021 and PI16/01511 grant; the "CIBER Enfermedades Respiratorias" Network (CIBERES); the "Spanish Society of Pneumology and Thoracic Surgery" (SEPAR) through grant 16/023; and the "Agència de Gestió d'Ajuts Universitaris i de Recerca" (AGAUR) through AD contract (2017 FI_B_00797). Funding information for this article has been deposited with the Crossref Funder Registry.

\section{References}

1 The Regional Office for Europe of the World Health Organization. The role of surgery in the treatment of pulmonary TB and multidrug- and extensively drug-resistant TB. Copenhagen, WHO Regional Office for Europe, 2014.

2 World Health Organization. Global tuberculosis report 2018. Geneva, World Health Organization, 2018.

3 World Health Organization. WHO consolidated guidelines on drug-resistant tuberculosis treatment. Geneva, World Health Organization, 2019.

4 World Health Organization. Implementing the End TB Strategy: the essentials. Geneva, World Health Organization, 2015.

5 Jones P. St George's Respiratory Questionnaire Manual. Saint George's, University of London, 2009. www. healthstatus.sgul.ac.uk/SGRQ_download/SGRQ\%20Manual\%20June\%202009.pdf

6 Jones PW, Quirk FH, Baveystock CM. The St George's Respiratory Questionnaire. Respir Med 1991; 85: Suppl. B, 25-31.

7 Jones PW, Quirk FH, Baveystock CM, et al. A self-complete measure of health status for chronic airflow limitation. The St George's Respiratory Questionnaire. Am Rev Respir Dis 1992; 145: 1321-1327.

8 Chang B, Wu AW, Hansel NN, et al. Quality of life in tuberculosis: a review of the English language literature. Qual Life Res 2004; 13: 1633-1642.

9 Brown J, Capocci S, Smith C, et al. Health status and quality of life in tuberculosis. Int J Infect Dis 2015; 32: 68-75.

10 Ferrer M, Villasante C, Alonso J, et al. Interpretation of quality of life scores from the St George's Respiratory Questionnaire. Eur Respir J 2002; 19: 405-413.

11 Jones PW. Interpreting thresholds for a clinically significant change in health status in asthma and COPD. Eur Respir J 2002; 19: 398-404.

12 Vashakidze SA, Kempker JA, Jakobia NA, et al. Pulmonary function and respiratory health after successful treatment of drug-resistant tuberculosis. Int J Infect Dis 2019; 82: 66-72.

13 Hertz D, Schneider B. Sex differences in tuberculosis. Semin Immunopathol 2019; 41: 225-237.

14 Vashakidze S, Despuig A, Gogishvili S, et al. Retrospective study of clinical and lesion characteristics of patients undergoing surgical treatment for pulmonary tuberculosis in Georgia. Int J Infect Dis 2017; 56: 200-207.

15 Byng-Maddick R, Noursadeghi M. Does tuberculosis threaten our ageing populations? BMC Infect Dis 2016; 16 119.

16 Chandrasekaran P, Saravanan N, Bethunaickan R, et al. Malnutrition: modulator of immune responses in tuberculosis. Front Immunol 2017; 8: 1-8.

17 Hoyt KJ, Sarkar S, White L, et al. Effect of malnutrition on radiographic findings and mycobacterial burden in pulmonary tuberculosis. PLoS ONE 2019; 14: 1-11.

18 Bhargava A, Chatterjee M, Jain Y, et al. Nutritional status of adult patients with pulmonary tuberculosis in rural Central India and its association with mortality. PLoS ONE 2013; 8: 1-11.

19 Leung CC, Yew WW, Chan CK, et al. Smoking adversely affects treatment response, outcome and relapse in tuberculosis. Eur Respir J 2015; 45: 738-745.

20 Kempker RR, Vashakidze S, Solomonia N, et al. Surgical treatment of drug-resistant tuberculosis. Lancet Infect Dis 2012; 12: 157-166.

21 Abdulelah J, Sulaiman SAS, Hassali MA, et al. Development and psychometric properties of a tuberculosis-specific multidimensional health-related quality-of-life measure for patients with pulmonary tuberculosis. Value Health Reg Issues 2015; 6: 53-59.

22 Sun Y, Yang Z, Wan C, et al. Development and validation of the pulmonary tuberculosis scale of the system of quality of life instruments for chronic diseases (QLICD-PT). Health Qual Life Outcomes 2018; 16: 137-146.

23 Boudioni M, McLaren S, Belling R, et al. Listening to those on the frontline: service users' experiences of London tuberculosis services. Patient Prefer Adherence 2011; 5: 267-277.

24 Hansel NN, Wu AW, Chang B, et al. Quality of life in tuberculosis: patient and provider perspectives. Qual Life Res 2004; 13: 639-652.

25 Odone A, Houben RMGJ, White RG, et al. The effect of diabetes and undernutrition trends on reaching 2035 global tuberculosis targets. Lancet Diabetes Endocrinol 2014; 2: 754-764.

26 Koethe JR, Von Reyn CF. Protein-calorie malnutrition, macronutrient supplements, and tuberculosis. Int J Tuberc Lung Dis 2016; 20: 857-863.

27 Kant S, Gupta H, Ahluwalia S. Significance of nutrition in pulmonary tuberculosis. Crit Rev Food Sci Nutr 2015 55: 955-963.

28 Frediani JK, Sanikidze E, Kipiani M, et al. Macronutrient intake and body composition changes during antituberculosis therapy in adults. Clin Nutr 2016; 35: 205-212.

29 Rieder H. Epidemiologic basis of tuberculosis control. Paris, International Union Against Tuberculosis and Lung Disease, 1999.

30 Zürcher $\mathrm{K}$, Ballif $\mathrm{M}$, Zwahlen $\mathrm{M}$, et al. Tuberculosis mortality and living conditions in Bern, Switzerland, 1856-1950. PLoS ONE 2016; 11: e0149195.

31 Martire LM, Helgeson VS. Close relationships and the management of chronic illness: associations and interventions. Am Psychol 2017; 72: 601-612.

32 World Health Organization. The End TB Strategy. Global strategy and targets for tuberculosis prevention, care and control after 2015. A67/11. Geneva, World Health Organization, 2014.

33 Lönnroth K, Glaziou P, Weil D, et al. Beyond UHC: monitoring health and social protection coverage in the context of tuberculosis care and prevention. PLoS Med 2014; 11: e1001693. 
34 Tola HH, Shojaeizadeh D, Garmaroudi G, et al. Psychological distress and its effect on tuberculosis treatment outcomes in Ethiopia. Glob Health Action 2015; 8: 29019.

35 Akinci B, Aslan GK, Kiyan E. Sleep quality and quality of life in patients with moderate to very severe chronic obstructive pulmonary disease. Clin Respir J 2018; 12: 1739-1746.

36 Scharf SM, Maimon N, Simon-Truval T, et al. Sleep quality predicts quality of life in chronic obstructive pulmonary disease. Int J Chron Obstruct Pulmon Dis 2010; 6: 1-12.

37 Vardar-Yagli N, Saglam M, Savci S, et al. Impact of sleep quality on functional capacity, peripheral muscle strength and quality of life in patients with chronic obstructive pulmonary disease. Expert Rev Respir Med 2015; 9: 233-239.

38 Chang $\mathrm{CH}$, Chuang LP, Lin SW, et al. Factors responsible for poor sleep quality in patients with chronic obstructive pulmonary disease. BMC Pulm Med 2016; 16: 118-125.

39 Sagwa EL, Ruswa N, Mavhunga F, et al. Adverse events and patients' perceived health-related quality of life at the end of multidrug-resistant tuberculosis treatment in Namibia. Patient Prefer Adherence 2016; 10: 2369-2377.

40 Sineke T, Evans D, Schnippel K, et al. The impact of adverse events on health-related quality of life among patients receiving treatment for drug-resistant tuberculosis in Johannesburg, South Africa. Health Qual Life Outcomes 2019; 17: 94-108. 